\title{
1 Engineering analysis with probability boxes: a review on computational methods
}

\author{
2 Matthias G.R. Faes ${ }^{\mathrm{a}, \mathrm{e}}$, Marco Daub ${ }^{\mathrm{b}, \mathrm{c}}$, Stefano Marelli ${ }^{\mathrm{b}}$, Edoardo Patellid,f, Michael Beer ${ }^{\mathrm{e}, \mathrm{f}, \mathrm{g}}$ \\ ${ }^{a}$ KU Leuven, Jan De Nayerlaan 5, 2860 Sint-Katelijne-Waver, Belgium. Email: matthias.faes@kuleuven.be \\ ${ }^{b}$ ETH Zürich, Stefano-Franscini-Platz 5, 8093 Zürich, Switzerland \\ ${ }^{c}$ Technical University of Munich, Arcistr. 21, 80333 Munich, Germany \\ ${ }^{d}$ Strathclyde University, Department of Civil and Environmental Engineering, \\ ${ }^{e}$ Institute for Risk and Reliability, Leibniz Universität Hannover, Callinstr. 34, 30167 Hannover, Germany \\ ${ }^{f}$ Institute for Risk and Uncertainty and School of Engineering, University of Liverpool, Peach Street, Liverpool L69 7ZF, \\ $U K$ \\ ${ }^{g}$ International Joint Research Center for Engineering Reliability and Stochastic Mechanics, Tongji University, 1239 Siping \\ Road, Shanghai 200092, P.R. China
}

\section{Abstract}

The consideration of imprecise probability in engineering analysis to account for missing, vague or incomplete data in the description of model uncertainties is a fast-growing field of research. Probability-boxes (p-boxes) are of particular interest in an engineering context, since they offer a mathematically straightforward description of imprecise probabilities, as well as allow for an intuitive visualisation. In essence, p-boxes are defined via lower and upper bounds on the cumulative distribution function of a random variable whose exact probability distribution is unknown. However, the propagation of p-boxes on model inputs towards bounds on probabilistic measures describing the uncertainty on the model responses is numerically still very demanding, and hence is subject of intensive research. In order to provide an overview on the available methods, this paper gives a state-of-the art review for the modelling and propagation of p-boxes with a special focus on structural reliability analysis.

Keywords: imprecise probability, p-boxes, literature review, reliability analysis, surrogate modelling

\section{Introduction}

Numerical models give an unparalleled insight into the response of the structure under consideration to a set of predefined loading conditions, and hence, allow for a largely virtualized design optimization workflow. Examples of such models include finite element models of structures or thermal systems, but also other numerical schemes aimed at approximating complex multi-physical systems from the nanoscopic to the largest possible level can be considered. However, despite the highly detailed numerical predictions that can be obtained, these results often do not achieve a satisfactory level of agreement with 'reality', i.e., the actual physical behaviour of the considered continuum in the effective operational environment. This discrepancy is caused by epistemic (reducible) and aleatory (caused by variation) uncertainty in the model. Usually, a distinction between model form and parametric uncertainty is made, where the 
former describes possibly unwarranted approximations of the mathematical description of reality, whereas the latter refers to discrepancies in the parameters of these models with respect to reality. This paper solely focuses on parametric uncertainties. In recent years, several highly performing methods based on stochastic analysis [1], fuzzy set theory and interval analysis [2] have been introduced in literature to account for these type of uncertainties in the model parameters $\boldsymbol{x}$. Also several authors compared the applicability of a selection of these techniques in applications such as Geotechnical engineering [3] or inverse uncertainty quantification for stochastic dynamics $[4,5]$.

\subsection{Probabilistic analysis}

Probabilistic analysis is a powerful and mature tool to deal with aleatory uncertainties in numerical analyses. In order to express aleatory uncertainty in the model parameters, they are usually modelled as random variables, denoted by $\boldsymbol{X}=\left(X_{1}, \ldots, X_{n_{x}}\right)$ with support domain $D_{\boldsymbol{X}} \subseteq D_{x}$. Their values are outcomes of a random experiment where a probability $P$ can be assigned to $\boldsymbol{X}$ taking a value within a specific measurable set that is a subset of $D_{\boldsymbol{X}}$. The probability that $\boldsymbol{X}$ is less than or equal to $\boldsymbol{x}$ is modelled as a joint cumulative distribution function (CDF) $F_{\boldsymbol{X}}(\boldsymbol{x})=P\left(X_{1} \leq x_{1}, \ldots, X_{n_{x}} \leq x_{n_{x}}\right)$ for $\boldsymbol{x} \in D_{\boldsymbol{X}}$. Its derivative is denoted by $f_{\boldsymbol{X}}$ and is known as the joint probability density function (PDF). Since the inputs of the model are represented by a random vector, it follows that the model responses become random variables $\boldsymbol{Y}$, which are distributed according to the (generally unknown) CDF $F_{\boldsymbol{Y}}$. Note that $F_{\boldsymbol{X}}$ and $F_{\boldsymbol{Y}}$ in general do not belong to the same family of distribution functions.

Let $\mathcal{M}$ represent a function that maps a set of $n_{x}$ input parameters $\boldsymbol{x} \in D_{x} \subseteq \mathbb{R}^{n_{x}}$, with $D_{x}$ a set of feasible input parameters (e.g., non-negative Young's moduli or contact stiffness values), to a set of $n_{y}$ output parameters $\boldsymbol{y} \in \mathbb{R}^{n_{y}}$ via following relationship:

$$
\boldsymbol{y}=\mathcal{M}(\boldsymbol{x}),
$$

where $\mathcal{M}$ may represent numerical model that provides a discretized approximation of the continuum physics that describe the modelling problem at hand. Usually, given $f_{\boldsymbol{X}}$, an analyst is then interested in computing the expected value of some random variable $\mathcal{H}(\boldsymbol{X})$, i.e., $E[\mathcal{H}(\boldsymbol{X})]$. Here, $E$ is the expected value operator and $\mathcal{H}$ is a function defined on $D_{\boldsymbol{X}}$. Typically, in this context, $\mathcal{H}$ is used to compute the $n$th central moments of $\boldsymbol{Y}$, with $n \in \mathbb{N}$. Hereto, $\mathcal{H}$ represents the component-wise exponentiation of the model responses $\boldsymbol{y}=\mathcal{M}(\boldsymbol{x})$, i.e., $\mathcal{H}(\boldsymbol{x})=\boldsymbol{y}^{n}$, or $\mathcal{H}(\boldsymbol{x})=\left(\boldsymbol{y}-\boldsymbol{\mu}_{\boldsymbol{Y}}\right)^{n}$ with $\boldsymbol{\mu}_{\boldsymbol{Y}}=E[\boldsymbol{Y}]$. In an engineering context, an analyst is mostly interested into whether their design, be it a structure, system or a complex network, will perform reliably given the uncertainties in their manufacturing and operating conditions. Usually, the probability of failure is estimated in this context to assess the reliability of their design. 
The probability of failure can be computed as $p_{\mathrm{f}}=P(\mathcal{M}(\boldsymbol{X}) \leq 0)$, where $\mathcal{M}$ with $n_{y}=1$ represents a performance function that indicates whether the design failed $(\mathcal{M}(\boldsymbol{x}) \leq 0)$ or not $(\mathcal{M}(\boldsymbol{x})>0)$ for $\boldsymbol{x} \in D_{\boldsymbol{X}}$. In this context, $\mathcal{H}(\boldsymbol{x})$ is defined as $\mathcal{H}(\boldsymbol{x})=I_{\mathcal{M}}(\boldsymbol{x})$ with $I_{\mathcal{M}}$ the indicator function that has value 1 in case $\mathcal{M}(\boldsymbol{x}) \leq 0, \boldsymbol{x} \in D_{\boldsymbol{X}}$, and 0 otherwise. Overall, the expected value of $\mathcal{H}(\boldsymbol{X})$ is determined by evaluating the integral of the following form:

$$
\mathcal{P}=\int_{D_{\boldsymbol{X}}} \mathcal{H}(\boldsymbol{x}) f_{\boldsymbol{X}}(\boldsymbol{x}) \mathrm{d} \boldsymbol{x}
$$

where the physical interpretation of $\mathcal{P}=E[\mathcal{H}(\boldsymbol{X})]$ depends on the definition of $\mathcal{H}$. For the remainder of the paper, the notation $\mathcal{H}$ is used to abstract the specific application (i.e., calculation of the moments or failure probability approximation) from the method that is being discussed. While at first sight it might be tempting to evaluate this integral using numerical quadrature schemes, such solutions become quickly unfeasible with respect to the non-linearity of the limit state function and/or the number of considered random variables [6], even though lower/upper bounds [7] or approximate solutions [8] exist in certain cases. In general, even integrating just the joint PDF (i.e., $\mathcal{H}=1$ ) is not so trivial by quadrature, as they tend to be extremely non-linear, especially when the random variables are highly correlated. Therefore, Eq. (2) is usually solved by asymptotic approximations [9] or advanced simulation methods such as subset simulation [10], directional importance sampling [11] or the probability density evolution method [12] in case of stochastic dynamics.

\subsection{Imprecise probabilistic analysis}

In most real-life applications, an analyst has only partial information about $F_{\boldsymbol{X}}$ or $f_{\boldsymbol{X}}$ due to the presence of epistemic uncertainty. This is a result of the often imprecise, diffuse, fluctuating, incomplete or vague nature of the available information. Moreover, the available information might be objective or subjective and consist of collected data (e.g., via experiments or data mining) and theoretical knowledge on the considered problem, but also expert opinions with different levels of trustworthiness [13]. Some illustrations of such situations can be found in the benchmark study presented in [14]. In engineering analysis, the main challenge is then to formulate suitable models that incorporate these various sources of data in an objective way, without introducing unwarranted conclusions and/or ignoring significant information to ensure that the calculated results do not deviate from reality. The class of imprecise probabilistic approaches attempts to solve this general problem and includes a plethora of different methods, including Bayesian methods $[15,16,17,18]$, random sets $[19,20,21]$, sets of probability measures [22], evidence theory-based methods (such as Dempster-Shafer Theory) $[23,24,25,26]$ and interval probabilities [27] of which probability bounds methods [28] and fuzzy stochastic methods [29, 30] are extensions. 


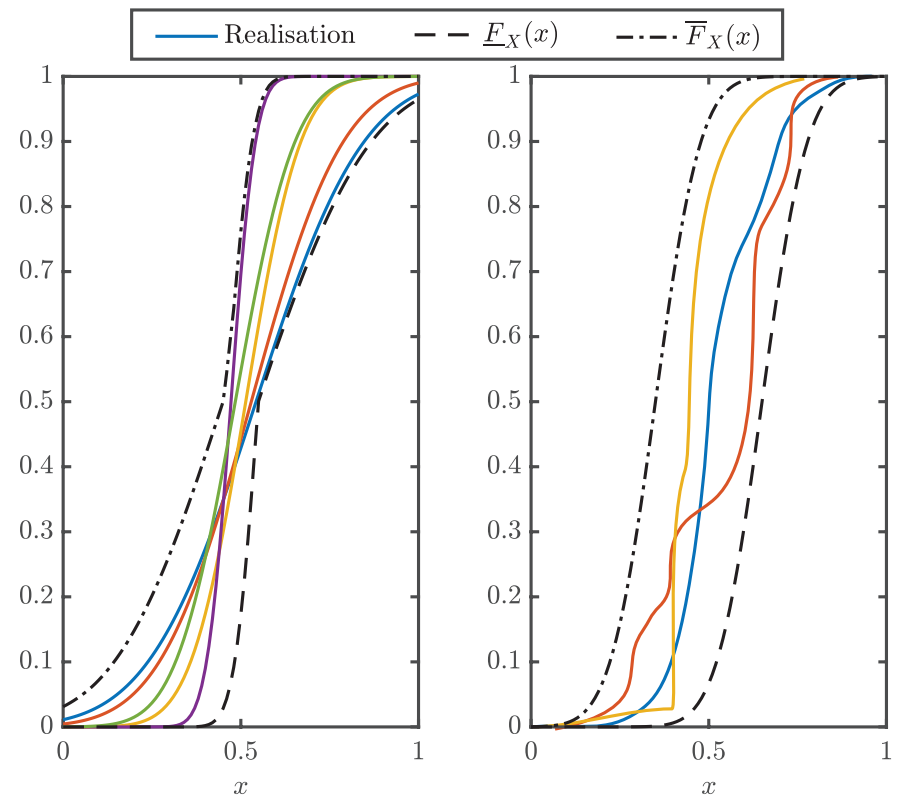

Figure 1: Illustration of parametric and distribution-free p-boxes. The black lines indicate the graphs of $\bar{F}_{X}$ and $\underline{F}_{X}$, being the bounds on the p-boxes. The colored lines illustrate a set of admissible distribution functions for $F_{X}$ that constitute the p-box.

Furthermore, a study of Monte Carlo methods for the general case of propagating imprecise probabilities is given for instance in [31] or [32]. Answering the question on which of these methods is the most appropriate method from this broad class of techniques is in general not possible as the most appropriate mathematical framework depends on the nature of the information that is available to the analyst. It should be noted that the application of the general framework of imprecise probability theory requires complex mathematical descriptions and methods. Furthermore, due to several restricting assumptions that are required, the methods are sometimes also very hard to translate to engineering practice. For a thorough treatment on the selection of the most appropriate method, the reader is referred to [13].

In many engineering applications, simplified imprecise probability models are often preferable for simpler utilization and representation. A popular representative thereof are probability-boxes (p-boxes), which provide a set of possible probability distributions for $F_{\boldsymbol{X}}$ bounded by a lower CDF $\underline{F}_{\boldsymbol{X}}$ and an upper CDF $\bar{F}_{\boldsymbol{X}}$. This type of credal set encompassing the unknown CDF is computationally efficient [33], easy to construct [34], and offers a simple graphical representation, see Fig. 1. This figure shows the two main types of p-boxes, being parametric and distribution-free p-boxes. Distribution-free p-boxes consider only the upper and lower CDF, and any CDF that complies with these bounds is admissible. Parametric p-boxes on the other hand impose additional constraints on admissible distribution functions, for instance by defining a family of distribution functions. A rigorous and more detailed definition of both types of p-boxes is given in Section 2.1.

Their simpler utilization and representation make the application of p-boxes particularly interesting 
for engineering analysis. However note that even with all their benefits over other, more general, imprecise probability models, computations involving p-boxes still require large computational budgets as they incorporate effectively a set of probability distributions that all need to be accounted for. Hence, advanced methods for p-box propagation have been subject to intense research over the past decades and various efficient methods addressing numerous applications of different complexity were proposed. This paper aims at giving an overview of a selection of promising approaches for the propagation of p-boxes in engineering analysis. This is complemented by an introduction to p-boxes showing their relationship to related imprecise probability models including their translation, and capabilities how to construct p-boxes based on given information.

\subsection{A guideline to read this paper}

Depending on the need of the reader, this paper can be used in several ways. For instance, a newcomer in the field of imprecise probabilities and/or p-boxes might use the entire manuscript to get the overall ideas on the methods, as well as obtain the references to recent key works in the field. In this case, it is recommended to consider all sections of the paper. On the other hand, an analyst that is knowledgeable with imprecise probabilities, but is unsure how to model them based on available data will gain most from the information in Section 3. Conversely, if an analyst is unsure which state-of-the-art propagation method is best applicable for their problem, they are kindly referred to Section 4 and the references therein included. To give the full overview; Section 2 describes the theoretical foundations of p-boxes and their analysis. Section 3 discusses the construction of p-boxes based on various sources of information. Section 4 highlights a selection of developments for the propagation of p-boxes, published during the last few years and ends with a summarizing table. Finally, Section 5 lists the conclusions of this paper.

\section{Probability boxes}

In the following two sections, the case $n_{x}=1$ is considered for notational simplicity. This is furthermore warranted since most engineering literature on the subject, as will be clear from Section 3, either considers the univariate case of $n_{x}=1$, or when $n_{x}>1$ full independence among all $X_{i}, i=1, \ldots, n_{x}$, with $F_{\boldsymbol{X}}(\boldsymbol{x})=\prod_{i=1}^{n_{x}} F_{X_{i}}\left(x_{i}\right), \boldsymbol{x} \in D_{\boldsymbol{X}}$. For more information on the general modeling of multivariate p-boxes including dependence, the reader is referred to $[35,36]$.

\subsection{Theoretical background}

The main idea of a p-box is that there exist an unknown CDF $F_{X}$ of the random variable $X$ for which only bounds can be provided. Thus, a p-box is described by a lower CDF $\underline{F}_{X} \in \mathbb{F}$ and an upper CDF $\bar{F}_{X} \in \mathbb{F}$, where $\mathbb{F}$ expresses the set of all CDFs on $D_{X} \subseteq \mathbb{R}$. These CDFs are collected as a pair 
$\left[\underline{F}_{X}, \bar{F}_{X}\right]$ which yields a set of possible CDFs $\left\{F_{X} \in \mathbb{F} \mid \underline{F}_{X}(x) \leq F_{X}(x) \leq \bar{F}_{X}(x), x \in D_{X}\right\}$ for the unknown CDF of $X$. The definition of a p-box corresponds to defining a lower probability $\underline{P}$ and upper probability $\bar{P}$ on events $\{X \leq x\}=(-\infty, x] \cap D_{X}$, i.e., $\underline{P}(X \leq x)=\underline{F}_{X}(x)$ and $\bar{P}(X \leq x)=\bar{F}_{X}(x)$ for $x \in D_{X}$, which yields a credal set of probability measures. Via the p-box framework, the epistemic uncertainty that comes for example from incomplete data on $F_{X}(x)$ is accounted for by assigning an interval $\left[\underline{F}_{X}(x), \bar{F}_{X}(x)\right]$ for each value of $x \in \mathbb{R}$, see [34]. In case sufficient high quality information over the entire range of possible values for $x$ is available to the analyst, $\left[\underline{F}_{X}(x), \bar{F}_{X}(x)\right]$ will be a tight interval, and the p-box will be close to a crisp (deterministic) distribution. Otherwise, when less information is available, the bounds may become wider to acknowledge weaker confidence in the results. In case no further assumptions are made concerning the set of possible CDFs, this type of p-box is also denoted a distribution-free p-box. This is the most general type of p-box, which allows for the highest flexibility when modelling parameters subject to aleatory and epistemic uncertainty, since any non-decreasing and right-continuous function that is consistent with these bounds is admissible. Indeed, it can be shown that crisp values, intervals and crisp probability distributions are all special cases of the distribution-free p-box [28]. As a final note, since distribution-free p-boxes are so general in their definition, also CDFs that are questionable from a physical perspective are explicitly included in the definition.

Besides distribution-free p-boxes, there are parametric p-boxes, which are described by a family of CDFs whose parameters $\theta_{i} \in \mathbb{R}$ are unknown up to the property that they must be contained within intervals $\left[\underline{\theta}_{i}, \bar{\theta}_{i}\right], i=1, \ldots, n_{\theta}$. These parameters describe specific distribution properties and are collected in the vector $\boldsymbol{\theta} \in \mathbb{R}^{n_{\theta}}$. The Cartesian product of the intervals is also denoted as $D_{\boldsymbol{\theta}}$, i.e., it holds $\boldsymbol{\theta} \in D_{\boldsymbol{\theta}}$. Hence, a parametric p-box yields the set of possible $\operatorname{CDFs}\left\{F_{X}(\cdot, \boldsymbol{\theta}) \in \mathbb{F} \mid \boldsymbol{\theta} \in D_{\boldsymbol{\theta}}\right\}$ for the unknown $\mathrm{CDF}$ of the random variable $X$. An example of a parametric p-box can be defined as the Gaussian distribution family with parameters $\boldsymbol{\theta}=(\mu, \sigma)$ contained in $D_{\boldsymbol{\theta}}=\left[\underline{\mu}_{X}, \bar{\mu}_{X}\right] \times\left[\underline{\sigma}_{X}, \bar{\sigma}_{X}\right]$. Parametric p-boxes have the property to clearly distinguish between aleatory uncertainty, represented by the distribution family, and epistemic uncertainty, represented by the intervals for the parameters $\boldsymbol{\theta}$. The upper and lower bounding CDFs of a parametric CDF can be computed as

$$
\begin{aligned}
& \underline{F}_{X}(x)=\min \left\{F_{X}(x, \boldsymbol{\theta}) \mid \boldsymbol{\theta} \in D_{\boldsymbol{\theta}}\right\}, \\
& \bar{F}_{X}(x)=\max \left\{F_{X}(x, \boldsymbol{\theta}) \mid \boldsymbol{\theta} \in D_{\boldsymbol{\theta}}\right\}
\end{aligned}
$$

for $x \in D_{X}$. Note that the distribution-free p-box defined by these bounds does not correspond to the parametric p-box as the latter one is more restrictive in general, i.e. there are CDF within these bounds not belonging to the family of the parametric p-box. Both types of p-boxes are illustrated in Fig. 1.

In order to account for more information about the shape of CDFs, such as an admissible distribu- 
tion family, symmetry, or about bounds on one or more statistical moments of $F_{X}$, a p-box can also be described by a quintuple $\left(\bar{F}_{X}, \underline{F}_{X}, \mu_{X}^{I}, \sigma_{X}^{I}, \mathcal{F}\right)$, see [13]. Here, the confidence interval of the mean value $\mu_{x}^{I} \subseteq[-\infty, \infty]$, the confidence interval $\sigma_{x}^{I} \subseteq[0, \infty]$ of the standard deviation, and the family of admissible $\mathrm{CDFs} \mathcal{F} \subseteq \mathbb{F}$ can be specified. Note that a distribution-free p-box can also be represented as a quintuple, noted $\left(\bar{F}_{X}, \underline{F}_{X},[-\infty,+\infty],[0, \infty], \mathbb{F}\right)$. Furthermore, the p-box framework was also recently extended to account for imprecision in stochastic processes by explicitly accounting for additional epistemic uncertainty in the process' autocorrelation structure [37, 38].

In the following subsections, the connection of p-boxes to some closely related uncertainty models for imprecise probabilities is demonstrated. This may help the reader in both understanding the similarities and differences between p-boxes and these models and converting them into p-boxes or vice versa.

\subsection{Hierarchical probabilistic models}

An alternative approach to deal with parametric p-boxes is to apply hierarchical probabilistic models. Following this approach, the epistemic uncertainty related to the parameters $\boldsymbol{\theta}$ of the $\mathrm{CDF} F_{X}(\cdot, \boldsymbol{\theta})$ are represented using a random variable $\Theta$ with distribution $F_{\Theta}$. On the one hand, hierarchical probabilistic models can be regarded as a special case of a p-box where intervals are used to bound possible values of $\boldsymbol{\theta}$. According to possibility theory, these intervals encode the set of all distribution functions bounded by the interval. As such, selecting a single distribution function out of this set introduces knowledge into the analysis that might not be fully objective. On the one hand, parametric p-boxes might be constructed using credible intervals from Bayesian methods along with hierarchical probabilistic models, see Section 3.4. In this case, p-boxes describe an excerpt of this modelling where the tails of $F_{\Theta}$ are neglected.

Using hierarchical probabilistic models, the effect of the epistemic uncertainty on the probabilistic measure under consideration depends on the applied propagation schemes. For instance, when reweighting schemes such as presented in $[39,40,41]$ are applied to infer the bounds, this is not problematic since they allow for a clear separation between aleatory and epistemic uncertainty. In these types of methods, the distribution $F_{\Theta}$ is a purely instrumental tool to determine a functional relationship between $\mathcal{P}$ and $\boldsymbol{\theta}$, the influence of which is integrated out of the result in later stages of the analysis, see Section 4 . However, when this single distribution is used to make strong inference on the bounds of $\mathcal{P}$, e.g., via sampling, this will lead to inherent bias on the results of the analysis.

\subsection{Random sets}

A p-box can be regarded as a special case of a random set, which has important implications for some of the propagation methods explained in Section 4. To see this, consider a probability space $\left(\Omega, \mathcal{F}_{\Omega}, P_{\Omega}\right)$ and a subset $\mathcal{K}_{X}$ of the power set of $D_{X} \subseteq X$. A random set $\Gamma_{X}$ is then a mapping 
$\Gamma_{X}: \Omega \rightarrow \mathcal{K}_{X}, \alpha \mapsto \Gamma_{X}(\alpha)$, where each $\Gamma_{X}(\alpha) \in \mathcal{K}_{X}, \alpha \in \Omega$, is called a focal element. When distributionfree p-boxes are defined as $\Gamma_{X}(\alpha)=\left[\bar{F}_{X}^{-1}(\alpha), \underline{F}_{X}^{-1}(\alpha)\right]$ for $\alpha \in \Omega$ and $\Omega=[0,1]$ with uniform probability distribution, they are a specific case of random sets, see [42]. Furthermore note that for finite $\mathcal{K}_{X}$, random sets correspond to a Demspter-Shafer structures, see also [42].

Since a random set is not capable of representing a single parameterized distribution family, a direct relationship with parametric p-boxes cannot be established [43, 44]. Conversion is possible however by first converting the parametric p-box into a distribution-free p-box, see Eq. (3) and (4). Moreover, $\Gamma_{X}(\alpha)$ can also be defined directly here via the inverse distributions of the family $F_{X}(\cdot, \boldsymbol{\theta}), \boldsymbol{\theta} \in D_{\boldsymbol{\theta}}$, i.e.,

$$
\Gamma_{X}(\alpha)=\left[\min _{\boldsymbol{\theta} \in D_{\boldsymbol{\theta}}} F_{X}^{-1}(\alpha, \boldsymbol{\theta}), \max _{\boldsymbol{\theta} \in D_{\boldsymbol{\theta}}} F_{X}^{-1}(\alpha, \boldsymbol{\theta})\right]
$$

as shown in [44].

\subsection{Fuzzy probabilities}

An extension to the p-box is provided by fuzzy probabilities, which allow for considering a fuzzy set of probability models, each having their own level of plausibility according to the available information [3]. According to this framework, the fuzzy membership function serves as an instrument to combine various plausible intervals $\left[\underline{F}_{X}^{\alpha}(x), \bar{F}_{X}^{\alpha}(x)\right], \alpha \in[0,1]$, for $x \in D_{X}$ to define distribution-free p-boxes in a single scheme, and hence, allows for assessing the sensitivity of the bounds $\underline{\mathcal{P}}^{\alpha}$ and $\overline{\mathcal{P}}^{\alpha}$ of $\mathcal{P}$. Indeed, sensitivities of $\mathcal{P}$ are found by considering the rate of change of the bounds on the interval with respect to the size of the input intervals represented in the fuzzy numbers. It holds $\left[\underline{F}_{X}^{\alpha_{i}}(x), \bar{F}_{X}^{\alpha_{i}}(x)\right] \subseteq\left[\underline{F}_{X}^{\alpha_{j}}(x), \bar{F}_{X}^{\alpha_{j}}(x)\right], x \in D_{X}$ and therefore $\left[\underline{\mathcal{P}}^{\alpha_{i}}, \overline{\mathcal{P}}^{\alpha_{i}}\right] \subseteq\left[\underline{\mathcal{P}}^{\alpha_{j}}, \overline{\mathcal{P}}^{\alpha_{j}}\right]$ for $0 \leq \alpha_{j} \leq \alpha_{i} \leq 1$. Furthermore, the concept can be also applied to parametric p-boxes, see [45]. Here, the fuzzy membership function is used to assign an $\alpha$-level to the parameters $\boldsymbol{\theta}$ of $F_{X}(\cdot, \boldsymbol{\theta})$. Then, the same analysis can be conducted as for distribution-free p-boxes. As the methods discussed further in the paper, which are developed for p-boxes, can always be applied to fuzzy probabilities in an $\alpha$-cut sense, the latter are not discussed in more detail.

\section{Construction of p-boxes for engineering analysis}

This section provides an overview how distribution-free and parametric p-boxes can be constructed based on given information. Here, a distinction is made between the three types of information: incomplete or imprecise distribution properties, datasets, or multiple sources of p-boxes. In the following, the focus is put on distribution-free p-boxes first. They are recommended when there is no knowledge in favour of a particular distribution family. If this information is available but the parameters $\boldsymbol{\theta}$ of $F_{X}(\cdot, \boldsymbol{\theta})$ 
are unknown, parametric p-boxes are preferred. A guide to find an appropriate construction method is provided in Table 1.

Furthermore note that distribution-free p-boxes can be always constructed as an approximation or an actual conversion of uncertainty models yielding lower and upper probabilities for events $X \leq x$, see Section 2. For a general introduction on the construction of p-boxes, the reader is referred to [34], where most of the approaches presented in the following are included. A comparison of selected methods can be found, e.g. in $[46,47]$.

Table 1: Overview of which sections in the paper provide an appropriate p-box construction method depending on the available information and p-box type.

\begin{tabular}{|l||c|c|}
\hline type & distribution-free p-box & parametric p-box \\
\hline incomplete distribution information & mean, variance, support: Sec. 3.1 & parameters: Sec. 3.4.1 \\
dataset & Sec. 3.2 & Sec. 3.4.2 \\
multiple sources & Sec. 3.3 & \\
\hline
\end{tabular}

\subsection{Incomplete distribution properties}

In the case that only a limited number of distribution properties are known, like its shape or support, moments, or quantiles, various methods to construct a p-box are available, see [34]. These methods use the information about the distribution properties to derive proper bounds on the distribution. Often, they are based on well-known statistical inequalities. In the following, three methods addressing the support $D_{X}$ and the first two moments of a random variable $X$ are presented exclusively. These assume limited but precisely known distribution properties.

\subsubsection{Support}

If only the support of a distribution is known, the interval $D_{X}=[\underline{x}, \bar{x}]$ can be used as a representation in case the support is bounded. This corresponds to a p-box described by two unit step functions $H_{\underline{x}}$ and $H_{\bar{x}}$ at its minimum and maximum values $\underline{x}$ and $\bar{x}$, i.e., $\underline{F}_{X}(x)=H_{\bar{x}}(x)$ and $\bar{F}_{X}(x)=H_{\underline{x}}(x)$ for $x \in D_{X}$.

\subsubsection{Mean and variance}

If the values of the mean $\mu_{X}$ and the variance $\sigma_{X}^{2}$ are known, the two-sided Chebyshev's inequality can be used to construct a p-box as described in [48], i.e.,

$$
\begin{aligned}
& \underline{F}_{X}(x)= \begin{cases}0 & \text { for } x<\mu+\sigma, \\
1-\frac{\sigma^{2}}{(x-\mu)^{2}}, & \text { for } x \geq \mu+\sigma,\end{cases} \\
& \bar{F}_{X}(x)= \begin{cases}\frac{\sigma^{2}}{(x-\mu)^{2}}, & \text { for } x<\mu-\sigma, \\
1, & \text { for } x \geq \mu-\sigma .\end{cases}
\end{aligned}
$$


for $x \in D_{X}$. Instead of Chebyshev's inequality, Cantelli's inequality is used to construct a p-box based on the mean and variance in [49].

\subsubsection{Mean, variance, and support}

If both its bounded support $D_{X}$ and its first two moments are known, the p-box bounds can be formulated as

$$
\begin{aligned}
& \underline{F}_{X}(x)= \begin{cases}0 & \text { for } x \leq \mu+\frac{\sigma^{2}}{\mu-\bar{x}}, \\
1-\frac{b(1+a)-c-b^{2}}{a} & \text { for } \mu+\frac{\sigma^{2}}{\mu-\bar{x}}<x<\mu+\frac{\sigma^{2}}{\mu-\underline{x}}, \\
\frac{1}{1+\frac{\sigma^{2}}{(x-\mu)^{2}}} & \text { for } \mu+\frac{\sigma^{2}}{\mu-\underline{x}} \leq x<\bar{x} \\
1 & \text { for } x \geq \bar{x}\end{cases} \\
& \bar{F}_{X}(x)= \begin{cases}0 & \text { for } x \leq \underline{x}, \\
\frac{1}{1+\frac{(x-\mu)^{2}}{\sigma^{2}}} & \text { for } \underline{x}<x \leq \mu+\frac{\sigma^{2}}{\mu-\bar{x}}, \\
1-\frac{b^{2}-a b+c}{1-a} & \text { for } \mu+\frac{\sigma^{2}}{\mu-\bar{x}}<x<\mu+\frac{\sigma^{2}}{\mu-\underline{x}} \\
1 & \text { for } x \geq \mu+\frac{\sigma^{2}}{\mu-\underline{x}}\end{cases}
\end{aligned}
$$

where $a=\frac{x-\underline{x}}{\bar{x}-\underline{x}}, b=\frac{\mu-\underline{x}}{\bar{x}-\underline{x}}, c=\frac{\sigma^{2}}{(\bar{x}-\underline{x})^{2}}$, see e.g. [50]. Eq. (8) and (9) are based on the one-sided Chebyshev's inequalities and are tighter compared to the bounds in Section 3.1.1 and 3.1.2.

\subsection{Dataset}

In case limited information about the probability distribution is available in form of a dataset $\mathcal{X} \subset$ $\mathbb{R}^{n_{x}}$, the properties that are used in the methods of Section 3.1 can be estimated. In order to account for this estimation however, these methods need to be slightly adapted to inform the p-boxes, as described in $[51,49]$ for the sample mean and sample variance. Moreover, there are also methods which do not require an estimation of distribution properties for a given dataset: the methods of Kolmogorov-Smirnoff confidence bounds and robust Bayes. They are widely used in literature and are described briefly below. Note that data-based methods generally do not provide absolute bounds for p-boxes due to their nature, e.g. by using a confidence level smaller than 1 to avoid conservatism.

\subsubsection{Distribution support estimation}

In case very few data-points are available, estimating the bounds of the support of the p-box might be the only option for an analyst. This estimation can for instance be based on worst-case likelihood estimation [52], potentially in combination with Bayesian approaches [53]. Scenario optimization [54] can also be used in this context to obtain bounds with a proven degree of robustness under mild assumptions. 


\subsubsection{Kolmogorov-Smirnoff confidence bounds}

Given a dataset $\mathcal{X}$ with $N$ samples, an empirical distribution $F_{\mathcal{X}}$ can be computed. Then, KolmogorovSmirnoff (K-S) confidence bounds for $F_{\mathcal{X}}$ define the bounds of a p-box as proposed in [34]. For $x \in D_{X}$, it holds

$$
\begin{aligned}
& \underline{F}_{X}(x)=\min \left(1, \max \left(0, F_{\mathcal{X}}(x)-D_{N}^{\alpha}\right)\right), \\
& \bar{F}_{X}(x)=\min \left(1, \max \left(0, F_{\mathcal{X}}(x)+D_{N}^{\alpha}\right)\right),
\end{aligned}
$$

where $D_{N}^{\alpha}$ is a K-S critical value at significance level $\alpha$ for a dataset with $N$ samples which can be found in $[55]$.

\subsubsection{Robust Bayes}

Furthermore, a p-box can be obtained by using robust Bayes methods, introduced by [56]. Here, the basic idea is to consider the parameters $\boldsymbol{\theta}$ also as random variables expressed in $\boldsymbol{\Theta}$ and to apply standard Bayesian inference to all plausible likelihood functions $L(\cdot, \mathcal{X})$ and all plausible prior distributions. Here, $f_{\Theta}$ denotes the PDF of the prior and $L(\cdot, \mathcal{X})$ is the likelihood of observing $\mathcal{X}$ depending on the incorporated distribution family $F_{X}(\cdot, \boldsymbol{\theta})$. This implies a class of posterior PDFs of $\boldsymbol{\Theta}$, denoted by $f_{\Theta}(\cdot \mid \mathcal{X})$, via Bayes theorem

$$
f_{\Theta}(\boldsymbol{\theta} \mid \mathcal{X})=\frac{L(\mathcal{X}, \boldsymbol{\theta})}{\int_{D_{\Theta}} L(\mathcal{X}, \boldsymbol{\theta}) f_{\Theta}(\boldsymbol{\theta}) \mathrm{d} \boldsymbol{\theta}} f_{\Theta}(\boldsymbol{\theta})
$$

and pairwise combination. Then, a p-box can be constructed by the envelope of all resulting CDFs using Bayesian point estimates, see [57], or credible intervals/regions like discussed in Section 3.4.2, see [46]. Moreover, a Bayesian pointwise approach that considers specific percentiles of all resulting CDFs can be used for the construction of a p-box as well, see [58].

\subsection{Aggregation of p-boxes}

In the methods above, the intention was to obtain a p-box based-on given information. If there are already $n_{\mathrm{p}}$ p-boxes $\left[\underline{F}_{X}^{(j)}, \bar{F}_{X}^{(j)}\right]$ available to describe a single quantity, aggregation methods can be used. In the following, three popular methods, namely the envelope, intersection, and mixture strategy, are reviewed. For further methods, the reader is once again referred to [34].

\subsubsection{Envelope and intersection}

If there are multiple p-boxes of which it at least one encompasses the unknown CDF of $X$, but there is no information which p-boxes really encompass it, the envelope strategy can be used. Here, an envelope 
p-box is defined as

$$
\begin{aligned}
& \underline{F}_{X}(x)=\min \left\{\underline{F}_{X}^{(j)}(x) \mid j=1, \ldots, n_{\mathrm{s}}\right\} \\
& \bar{F}_{X}(x)=\max \left\{\bar{F}_{X}^{(j)}(x) \mid j=1, \ldots, n_{\mathrm{s}}\right\}
\end{aligned}
$$

for $x \in D_{X}$. This corresponds to a conservative modelling. Opposite to the envelope strategy, there is the intersection strategy for which all available p-boxes are considered as reliable. Here, the intersection of all p-boxes is used, see [34]. For this strategy, the min and max operators in Eq. (13) and (14) are exchanged.

\subsubsection{Mixture}

If there are multiple p-boxes which were constructed for specific situations that suffer under variability, the mixture strategy can be used for the condensation in a single p-box. Here, the idea is to use weights $w_{j}>0$ with $W=\sum_{j=1}^{n_{\mathrm{s}}} w_{j}$ to express the relative frequencies. Then, the mixture p-box is defined as

$$
\begin{aligned}
& \underline{F}_{X}(x)=\frac{1}{W} \sum_{j=1}^{n_{\mathrm{s}}} w_{j} \underline{F}_{X}^{(j)}(x), \\
& \bar{F}_{X}(x)=\frac{1}{W} \sum_{j=1}^{n_{\mathrm{s}}} w_{j} \bar{F}_{X}^{(j)}(x)
\end{aligned}
$$

for $x \in D_{X}$. A special case are even weights, e.g., $w_{j}=1, j=1, \ldots, n_{\mathrm{s}}$ with $W=n_{\mathrm{s}}$, which correspond to an arithmetic averaging of the p-boxes.

\subsection{Parametric p-box construction}

In order to construct a parametric p-box, the distribution family must be known. Hence, the problem of constructing a p-box reduces to establishing bounding intervals for the corresponding parameters $\boldsymbol{\theta}$ of $F_{X}(\cdot, \boldsymbol{\theta})$. Usually, these intervals are assumed or estimated for a given dataset, see the methods below. Note that all methods to obtain a parametric p-box can be also used to build a distribution-free p-box by using Eq. (3) and (4) which yield the envelope of the parametric p-box.

\subsubsection{Bounds on distribution parameters}

In case bounds on the parameters $\boldsymbol{\theta}$ are available, e.g., from expert knowledge, the intervals for these parameters can be specified directly. For lower bounds $\underline{\theta}_{i}$ and an upper bounds $\bar{\theta}_{i}, i=1, \ldots, n_{\theta}$ their domain is denoted by $D_{\theta}$ (see Section 2.1). If there are $n_{\mathrm{s}}$ sources that provide different intervals, aggregation methods similar to Section 3.3 could be used, e.g., an envelope of all candidate domains $D_{\theta}^{(j)}$, where $\underline{\theta}_{i}=\min \left\{\underline{\theta}_{i}^{(j)} \mid j=1, \ldots, n_{\mathrm{s}}\right\}$ and $\bar{\theta}_{i}=\max \left\{\bar{\theta}_{i}^{(j)} \mid j=1, \ldots, n_{\mathrm{s}}\right\}, i=1, \ldots, n_{\theta}$. 


\subsubsection{Dataset}

Given a dataset $\mathcal{X}$, there are several methods to obtain interval estimates for the parameters $\boldsymbol{\theta}$ of $F_{X}(\cdot, \boldsymbol{\theta})$. Popular methods comprise confidence intervals from classical statistics, which cover the unknown, but deterministic parameters with a probability $\alpha$, or credible intervals from Bayesian statistics, in which the random vector $\Theta$, representing the parameters of the CDF, can be found with a probability $\alpha$, see [59] for further information on their computation. Note that in general independence between the parameters $\boldsymbol{\theta}$ needs to be assumed for $n_{\theta}>1$ in order to obtain interval regions.

\section{Propagation methods for p-boxes}

This section discusses commonly applied numerical schemes for propagating p-boxes towards bounds on the $n^{t h}$ central moment of the model response to a load and/or the probability of failure of the designed structure, system or complex network. In the case where $\boldsymbol{X}$ is represented as a p-box, a direct calculation of $\mathcal{P}$, as introduced in Eq. (2), is no longer possible since a set of PDFs that are consistent with the definition of the p-box has to be considered. Indeed, the consideration of a set of $f_{\boldsymbol{X}}$ causes the probabilistic measure $\mathcal{P}$ to become set-valued, too. The solution of this problem requires dedicated numerical procedures, which are described in the proceeding sections.

\subsection{Double loop approaches}

In case $\boldsymbol{X}$ represents a distribution-free p-box, the lower and upper bounds $\underline{\mathcal{P}} \leq \mathcal{P} \leq \overline{\mathcal{P}}$ can be obtained by solving the following optimization problems:

$$
\underline{\mathcal{P}}=\min _{f_{\boldsymbol{X}}} \int_{D_{X}} \mathcal{H}(\boldsymbol{x}) f_{\boldsymbol{X}}(\boldsymbol{x}) \mathrm{d} \boldsymbol{x}
$$

and:

$$
\overline{\mathcal{P}}=\max _{f_{\boldsymbol{X}}} \int_{D_{X}} \mathcal{H}(\boldsymbol{x}) f_{\boldsymbol{X}}(\boldsymbol{x}) \mathrm{d} \boldsymbol{x} .
$$

Note that these optimization problems are potentially very complicated since the optimization has to be carried out over the set of all possible $f_{\boldsymbol{X}}$ consistent with the definition of the p-box. Hence, this constitutes a non-convex, discontinuous optimization problem, which are notoriously difficult so solve exactly. In certain cases, tighter bounds on $\mathcal{P}$ can be obtained by means of linear programming, without having to construct the probability bounds of the input random variables $[60,61]$.

A first approach to simplify the optimization problems is to slice the p-box in order to transform the above problem into the propagation of a large number of intervals, each having a corresponding probability mass, which are then propagated through $\mathcal{M}$ to infer bounds on $\mathcal{P}$. The propagation of intervals is a well-understood problem in the context of uncertainty propagation [2]. However, following 
this approach the required number of evaluations of Eq. (1) scales exponentially with $n_{x}$ [62]. This led to the development of methods such as interval Monte Carlo simulation [62] or interval-Quasi Monte Carlo simulation [63]. These methods manage to break this exponential scaling of the computational cost by bounding $\mathcal{P}$ using following formulations:

$$
\begin{aligned}
& \underline{\mathcal{P}}=\frac{1}{n} \sum_{k=1}^{n} \overline{\mathcal{H}}\left(\boldsymbol{r}_{k}\right), \\
& \overline{\mathcal{P}}=\frac{1}{n} \sum_{k=1}^{n} \underline{\mathcal{H}}\left(\boldsymbol{r}_{k}\right)
\end{aligned}
$$

with $\overline{\mathcal{H}}\left(\boldsymbol{r}_{k}\right)$ and $\underline{\mathcal{H}}\left(\boldsymbol{r}_{k}\right)$ defined as:

$$
\begin{aligned}
& \overline{\mathcal{H}}\left(\boldsymbol{r}_{k}\right)=\max \left\{\mathcal{H}(\boldsymbol{x}) \mid \bar{F}_{\boldsymbol{X}}^{-1}\left(\boldsymbol{r}_{k}\right) \leq \boldsymbol{x} \leq \underline{F}_{\boldsymbol{X}}^{-1}\left(\boldsymbol{r}_{k}\right)\right\}, \\
& \underline{\mathcal{H}}\left(\boldsymbol{r}_{k}\right)=\min \left\{\mathcal{H}(\boldsymbol{x}) \mid \bar{F}_{\boldsymbol{X}}^{-1}\left(\boldsymbol{r}_{k}\right) \leq \boldsymbol{x} \leq \underline{F}_{\boldsymbol{X}}^{-1}\left(\boldsymbol{r}_{k}\right)\right\} .
\end{aligned}
$$

The parameters $\boldsymbol{r}_{k}, j=1, \ldots, N$ are realisations of a sample of $N$ independent and identically distributed (i.i.d.) random variables according to a multivariate standard uniform distribution. As is clear from these equations, a large number of model evaluations is still required to estimate of the bounds on $\mathcal{P}$ with sufficiently small variance, especially since an interval propagation problem (Eq. (21)) has to be solved for each $\boldsymbol{r}_{k}$. Note that in the general case, this interval problem has to be solved using global optimization approaches to accommodate possible non-convexity in $\mathcal{M}$ with respect to $\boldsymbol{x}$ and/or $\boldsymbol{\theta}$ [64]. Further improvement in computational efficiency can be obtained by resorting to efficient interval propagation schemes such as those based on Bernstein polynomials [65, 66], Cauchy deviates [28] (as recently applied in [67] and [68]), the transformation method [69] or Taylor series expansion methods [70, 71]. Further improvements in terms of efficiency can be obtained by using saddle-point approximations, as introduced in [72]. A more general version of the interval Monte Carlo approach was introduced by Alvarez in $[42,73]$ based on random sets (see also subsection 2.3). The main advantage of considering the full random set is that this representation is more general, and hence, intervals and Dempster-Shafer structures can be considered as well in the same framework [74]. Furthermore, the framework allows for including efficient sampling schemes, such as e.g., subset simulation [74].

In the case of parametric p-boxes, the extrema represented by Eq.(17) and Eq.(18) can be determined directly since the set of all possible $f_{\boldsymbol{X}}$ is readily parameterized. In this case, for each realisation of these parameters of $f_{\boldsymbol{X}}$, a reliability problem is solved, for instance for linear limit-state functions using FORM as presented in [75], or in more general cases using simulation methods. Using simulation methods, even in the simplest case where the p-box describes a set of possible $f_{\boldsymbol{X}}$ by means of interval-valued statistical 
moments, such calculation can be prohibitively demanding from a numerical standpoint. On the one hand, the calculation of the failure probability for a fixed value of the parameters associated with the stochastic process is quite costly. On the other hand, solving the associated optimization problems in this simple case is far from trivial, as it constitutes a double loop problem, where the inner loop comprises probability estimations, leading to possibly non-smooth behaviour of the objective function due to the inherent variance on the estimator of $\mathcal{P}$. Hence, apart from considering near-trivial simulation models, the propagation of p-box-valued parameters towards the bounds on the probabilistic measure $\mathcal{P}$ is computationally intractable. Note that in some very specific cases, analytical solutions are also available [76].

\subsection{Decoupling methods}

The class of decoupling methods aims at decoupling the double loop, presented in Eqs. (17) and (18) by separating the propagation of aleatory and epistemic uncertainties. This class of methods includes techniques based on importance sampling and operator norm theory. Both methods are restricted to parametric p-boxes, more precisely, p-boxes that are constructed by defining some parameters $\boldsymbol{\theta}$ of the distribution $F_{\boldsymbol{X}}(\boldsymbol{x} \mid \boldsymbol{\theta})$ to be interval valued.

\subsubsection{Importance sampling-based methods}

The core idea of importance sampling based methods is to propagate a single, well-chosen realisation $\hat{f}_{\boldsymbol{X}}$ of a parameterized p-box (where $\hat{f}_{\boldsymbol{X}}$ is optimal with respect to a predefined measure), and reweigh the obtained samples of $\boldsymbol{y}$ to infer bounds on $\mathcal{P}$.

A first such method is Extended Monte Carlo simulation, as introduced by [39], which is applicable to the propagation of parameterized p-boxes subjected to epistemic uncertainty in their first two moments, as well as the probability of failure. As a first step, the parameters $\boldsymbol{\theta}$ of the p-box, which account for $\mu_{x}$ and $\sigma_{x}$ in the quintuple description, are represented by a subjective probability model $f_{\boldsymbol{\Theta}}(\boldsymbol{\theta})=\prod_{i=1}^{n_{\theta}} f_{\Theta_{i}}\left(\theta_{i}\right)$. Then, a local estimation for $\mathcal{P}$, being $\hat{p}_{\mathrm{f}}$, is derived as:

$$
\hat{\mathcal{P}}(\boldsymbol{\theta})=\frac{1}{N} \sum_{k=1}^{N} \mathcal{H}\left(\boldsymbol{x}_{k}\right) \frac{f_{\boldsymbol{X}}\left(\boldsymbol{x}_{k} \mid \boldsymbol{\theta}\right)}{f_{\boldsymbol{X}}\left(\boldsymbol{x}_{k} \mid \boldsymbol{\theta}^{*}\right)}
$$

which is an unbiased estimator, but highly affected by the selection of $\boldsymbol{\theta}^{*}$. 'Local' in this context denotes that the estimator is derived for a fixed value of $\boldsymbol{\theta}$ inside its support $\boldsymbol{\theta}^{I}$. This fixed value, $\boldsymbol{\theta}^{*}$, should be selected such that it minimizes the variance on the estimator $\hat{\mathcal{P}}(\boldsymbol{\theta})$ [77], similarly to conventional Importance Sampling, as:

$$
\boldsymbol{\theta}^{*}=\operatorname{argmin} \int_{D_{\boldsymbol{\theta}}} T\left(\boldsymbol{\theta}, \boldsymbol{\theta}^{*}\right) f_{\boldsymbol{\Theta}}(\boldsymbol{\theta}) \mathrm{d} \boldsymbol{\theta}
$$


with $T\left(\boldsymbol{\theta}, \boldsymbol{\theta}^{*}\right)=V\left[\mathcal{H}(\boldsymbol{X}) f_{\boldsymbol{X}}(\boldsymbol{X} \mid \boldsymbol{\theta}) / f_{\boldsymbol{X}}\left(\boldsymbol{X} \mid \boldsymbol{\theta}^{*}\right)\right]$ and $V$ is the variance operator with respect to $f_{\boldsymbol{X}}(\cdot$ $\left.\boldsymbol{\theta}^{*}\right)$. The global version of this approach is based on realizations $\left(\boldsymbol{x}_{k}, \boldsymbol{\theta}_{k}\right), k=1, \ldots, N$ of a joint sample distributed according to a joint $\operatorname{PDF} f_{\boldsymbol{X}, \boldsymbol{\Theta}}$. The estimator $\hat{\mathcal{P}}(\boldsymbol{\theta})$ is in this case expressed as:

$$
\hat{\mathcal{P}}(\boldsymbol{\theta})=\frac{1}{N} \sum_{k=1}^{N} \mathcal{H}\left(\boldsymbol{x}_{k}\right) \frac{f_{\boldsymbol{X}}\left(\boldsymbol{x}_{k} \mid \boldsymbol{\theta}\right)}{f_{\boldsymbol{X}}\left(\boldsymbol{x}_{k} \mid \boldsymbol{\theta}_{k}\right)}
$$

where $\boldsymbol{x}_{k}$ and $\boldsymbol{\theta}_{k}$ are generated by applying the correct inverse probabilistic transform to the corresponding variables of a multivariate standard uniform distribution. The global estimator gives a better estimation of $\mathcal{P}$ over the entire support of $\boldsymbol{\theta}$, at the cost of lower accuracy around $\boldsymbol{\theta}^{*}$ and a higher computational cost, since in this case, also convergence in terms of the effect of $\boldsymbol{\theta}$ has to be ensured.

An alternative optimal sampling density to propagate parameterized p-boxes following a reweighted sampling scheme was proposed by [78, 79]. Following the approach of $[78,79]$, the optimal density should obtained by minimizing the total expected squared Hellinger distance between $f_{\boldsymbol{X}}(\cdot \mid \boldsymbol{\theta})$ and the optimal sampling density $f_{\boldsymbol{X}}\left(\cdot \mid \boldsymbol{\theta}^{*}\right)$ under an isoperimetric constraint that ensures that the derived optimal sampling density is a valid density function. The main difference with optimal sampling density presented in Eq. (24) is that this approach is not aimed at minimizing the variance, but rather that the sampling density is as close as possible to the target density.

\subsubsection{Advanced Line Sampling}

As an alternative decoupling strategy to deal with p-box valued uncertainty, Advanced Line Sampling was recently introduced [80]. Opposed to 'conventional' line sampling [81], this approach adaptively looks for the so-called important direction in standard normal space. Furthermore, due to this adaptive refinement, the same important direction can be used for the entire p-box analysis. Additionally, the method allows for reusing samples that are generated within the inner loop to be re-used during other iterations of the outer loop, significantly increasing the computational efficiency [80]. Based on these properties, a gain in computational efficiency of a factor of 4 over regular line-sampling approaches can be obtained, as reported in [80].

\subsubsection{Operator norm theory}

Operator norm theory provides an alternative pathway to decouple the double loop in Eq. (17) and (18), as presented first in [82] for the case of the class of linear models $\mathcal{M}$. In case an affine formulation of the imprecise random variables in terms of their parameters is possible, the propagation of the imprecise stochastic load can be performed in a two-step procedure. First, the values of the epistemic parameters that yield an extremum for $\mathcal{P}$ are determined by maximizing/minimizing the operator norm. 
436

Specifically, the operator norm is computed over the product of the linear mapping provided by the numerical model $\mathcal{M}$ with a basis $\boldsymbol{B}$ that represents the auto-correlation of the load on the model:

$$
\boldsymbol{\theta}^{*}=\underset{\boldsymbol{\theta} \in \boldsymbol{\theta}^{I}}{\operatorname{argmin}}\|\boldsymbol{A}(\boldsymbol{\theta})\|_{p^{(1)}, p^{(2)}}
$$

$$
\boldsymbol{\theta}^{\bar{*}}=\underset{\boldsymbol{\theta} \in \boldsymbol{\theta}^{I}}{\operatorname{argmax}}\|\boldsymbol{A}(\boldsymbol{\theta})\|_{p^{(1)}, p^{(2)}}
$$

with $\boldsymbol{A}=\mathcal{M} \boldsymbol{B}$, where $\boldsymbol{B}$ can for instance be determined following the well-known Karhunen-Loève expansion [82]. The operator norm $\|\boldsymbol{A}\|_{p^{(1)}, p^{(2)}}$ is generally defined as [83]:

$$
\|\boldsymbol{A}\|_{p^{(1)}, p^{(2)}}=\inf \left\{c \geq 0:\|\boldsymbol{A} \boldsymbol{v}\|_{p^{(1)}} \leq c \cdot\|\boldsymbol{v}\|_{p^{(2)}} \quad \forall \boldsymbol{v} \in \mathbb{R}^{n_{K L}}\right\}
$$

and gives a measure for how much $\boldsymbol{A}$ lengthens vector $\boldsymbol{v}$ in the maximum case. The practical calculation of the operator norm is case dependent. For instance, when considering first excursion problems $[84,85,86]$, i.e., $\mathcal{P} \equiv p_{\mathrm{f}}$, the selection of $p^{(1)} \rightarrow \infty$ and $p^{(2)}=2$ has been found to be a good choice [87]. In this case, the operator norm corresponds to the maximum $\mathcal{L}_{2}$ norm of a row of $\boldsymbol{A}$ [83]. Then, two failure probabilities, corresponding to $p_{\mathrm{f}}\left(\boldsymbol{\theta}^{*}\right)$ and $p_{\mathrm{f}}\left(\boldsymbol{\theta}^{\bar{*}}\right)$ have to be computed to determine the bounds on $\mathcal{P}$. As such, the double loop is effectively replaced by two deterministic optimizations and two crisp reliability estimations. Gains in computational efficiency with several orders of magnitude have been reported [82, 87]. The main drawback of the method is the limited scope, since the approach is only applicable to uncertain linear models with epistemic uncertain structural parameters, subjected to imprecisely defined load conditions.

\subsection{Surrogate modelling for p-boxes}

Surrogate models approximate well-selected 'regions' of $\mathcal{M}$ by a computationally more efficient surrogate model $\hat{\mathcal{M}}(\cdot \mid \boldsymbol{a})$. For instance, in the specific case of reliability analysis, $\hat{\mathcal{M}}(\cdot \mid \boldsymbol{a})$ is designed to be highly accurate in the region around the limit state function (i.e., $\mathcal{M}(\boldsymbol{x})=0$. This surrogate $\hat{\mathcal{M}}$, which is parameterized by a vector $\boldsymbol{a} \in \mathbb{R}^{n_{a}}$, is usually trained by means of a set of training data $\left\{\left(\boldsymbol{x}_{i}, \boldsymbol{y}_{i}\right) \mid i=1, \ldots, N\right\}$ via a supervised learning approach as to minimize the discrepancy between $\hat{\boldsymbol{y}}_{i}=\hat{\mathcal{M}}\left(\boldsymbol{x}_{i} \mid \boldsymbol{a}\right)$ and $\boldsymbol{y}_{i}$, according to a predefined measure (e.g., in an $L_{2}$ sense). These training data are generated either a priori (e.g., in case of sensitivity analysis) or enriched following active learning approaches [88, 89], which is most commonly applied in the field of reliability analysis. Examples of such maps to represent $\hat{\mathcal{M}}$ that have been used in the context of propagating p-boxes include Gaussian process models [90] (also known as Kriging), polynomial response surface models [91] or techniques based on Taylor series expansions [92]. Also adaptive schemes based on Kriging have been introduced in literature [57] that are applicable to both parametric and distribution-free p-boxes. In this section, 
three classes of methods are explained in detail that are highly promising from an engineering point of view due to their 'black-box' nature (i.e., they require no interaction with the inner operations of $\mathcal{M}$ ), theoretical implications and numerical efficiency. Note that in essence, each type of surrogate model can be used in combination with a double-loop approach since they are very cheap to evaluate. The selection of the appropriate type of surrogate model in fact only depends on $\mathcal{M}$.

\subsubsection{Polynomial Chaos Expansions Er Kriging models}

Polynomial chaos expansion (PCE) and Kriging are two widely used surrogate modelling techniques that approximate $\mathcal{M}$ via intricate regression schemes. In general, PCE and Kriging have different fields of application in the propagation of uncertainties. On the one hand, if the analyst is interested in propagating uncertainty in general (e.g., when $\mathcal{H}(\boldsymbol{x})=\left(\boldsymbol{y}-\boldsymbol{\mu}_{\boldsymbol{Y}}\right)^{n}$ ) PCE generally is better suited. Conversely, when considering reliability analysis, Kriging approaches are generally more performing since they allow for performing active learning [89, 93, 94], even though active learning approaches for PCE have also been introduced [95].

A sparse PCE surrogate model is given by:

$$
\hat{\mathcal{M}}(\boldsymbol{x} \mid \boldsymbol{a})=\sum_{\alpha \in \mathcal{A}} a_{\alpha} \boldsymbol{\phi}_{\alpha}(\boldsymbol{x})
$$

where $\phi_{\alpha}$ are multivariate orthonormal polynomials and $\mathcal{A} \subset \mathbb{N}^{n_{x}}$ is a finite set of multi-indices that is obtained by sparse decomposition. In [96], distribution-free p-boxes are propagated in a two-level approach in which first $\mathcal{M}$, and second $\underline{\mathcal{M}}$ and $\overline{\mathcal{M}}$ (in the sense of Eq (21) and (22)) are substituted using sparse PCE. The training set is generated for an auxiliary input vector $X$ and least angle regression (LARS) is used for training. In case of parametric p-boxes, it is proposed in [97] to model the sparse PCE coefficients $a_{\alpha}$ as quadratic polynomial functions of the parameters $\boldsymbol{\theta}$ of the p-box and using a double-loop sampling for the propagation.

Whereas PCE methods focus on the global behaviour of $\mathcal{M}$ and are therefore suitable for a general propagation of p-boxes, Kriging methods focus on a local behaviour of $\mathcal{M}$ and are therefore often preferred for reliability analysis. Indeed, in this context, a high accuracy in the vicinity where $\{\mathcal{M}=0\}$ is especially crucial. Using Kriging, a surrogate $\hat{\mathcal{M}}$ for the limit-state function is considered to be a realization of a Gaussian process. It is:

$$
\hat{\mathcal{M}}(\boldsymbol{x} \mid \boldsymbol{a})=\boldsymbol{\beta}_{\boldsymbol{a}}^{\mathrm{T}} \boldsymbol{\psi}(\boldsymbol{x})+Z_{\boldsymbol{a}}(\boldsymbol{x}, \omega),
$$

where the first term, consisting of coefficients $\boldsymbol{\beta}_{\boldsymbol{a}}$ and regression functions $\boldsymbol{\psi}$, is the mean value of the process, and the second term is a zero-mean, stationary Gaussian process, characterized by a variance and an auto-correlation function depending on $\boldsymbol{a}$. Similar to above, a two-level approach in which first $\mathcal{M}$, 
and second $\underline{\mathcal{M}}$ and $\overline{\mathcal{M}}$ are substituted is considered for distribution-free p-boxes in [57]. Here, adaptive Kriging Monte Carlo simulation (AK-MCS) is used for an accurate estimation of the failure probabilities and random slicing is used to obtain $\underline{\mathcal{P}}$ and $\overline{\mathcal{P}}$, see Eq. (19) and (20). Also in [57], a failure probability $\mathcal{P}(\boldsymbol{\theta})$ which depends on the parameters $\boldsymbol{\theta}$ is estimated via AK-MCS and efficient global optimization (EGO) for parametric p-boxes. A similar, but more detailed, Kriging-based procedure for parametric p-boxes is also described in [98].

\subsubsection{High-dimensional model representation based methods}

The Extended Monte Carlo framework, as introduced in Section 4.2.1 allows for propagating parametrized p-boxes by a single probabilistic simulation and a reweighting step. Nonetheless, still a considerable number of evaluations of $\mathcal{M}$ are required, which might impede practical applications. Therefore, in [39], both the local and global Extended Monte Carlo methods were integrated with a high-dimensional model representation (HDMR) decomposition of $\mathcal{M}$ as a surrogate modelling strategy. Following a HDMR deceomposition, $\mathcal{P}$ can be represented as:

$$
\begin{aligned}
\mathcal{P}(\boldsymbol{\theta})=p_{\mathrm{f}, 0} & +\sum_{i=1}^{n_{\theta}} p_{\mathrm{f}, i}\left(\theta_{i}\right)+\sum_{1 \leq i<j \leq d} p_{\mathrm{f}, i j}\left(\left[\theta_{i}, \theta_{j}\right]\right)+\ldots \\
& +p_{\mathrm{f}, 12 \ldots n_{\theta}}(\boldsymbol{\theta}) .
\end{aligned}
$$

Note that HDMR decompositions are more widely applicable than to represent $\mathcal{P}$. In the context of propagating p-boxes, in [39], it is proposed to apply a cut-HDMR strategy in combination with the local Extended Monte Carlo Method, allowing for a rigorous estimation of the variances of the estimators, as well as an estimation of the sensitivity of the parameters in $\boldsymbol{\theta}$. Similarly, it is proposed to perform a Random Slicing HDMR decomposition in combination with the Global Method. For the details concerning the implementation of these techniques, as well as the corresponding proofs, the reader is referred to [39]. These methods were recently also extended to be applied in combination with Line Sampling in [99].

An alternative application of the Sobol-Hoeffding decomposition in the context of propagating imprecise probabilities through numerical models is given by [100]. In [100], the authors apply a fuzzy probabilistic approach in the study of designing cylindrical shells under geometric imperfections, which are modelled as a random field. Specifically, imprecision in the auto-correlation structure of the random field is accounted for by means of fuzzy arithmetic, and the $\mathrm{S}-\mathrm{H}$ decomposition is applied to speed up the corresponding $\alpha$-level optimization. 


\subsubsection{Interval predictor models}

An interval predictor model (IPM), as introduced in [101], is a type of surrogate model that approximates $\mathcal{M}$ by means of an interval-valued map $\hat{\mathcal{M}}_{I}(\cdot, \boldsymbol{\theta}): \mathbb{R}^{n_{x}} \rightarrow \mathbb{I R}$, where $\mathbb{I}$ is the set of all intervals in $\mathbb{R}$. This map can be constructed with a minimal number of assumptions on the mapping provided by $\mathcal{M}$. Specifically, $\hat{\mathcal{M}}_{I}(\boldsymbol{x}, \boldsymbol{\theta})$ given by:

$$
\hat{\mathcal{M}}_{I}(\boldsymbol{x}, \boldsymbol{\theta})=\left\{y=\boldsymbol{\theta}^{\mathrm{T}} \boldsymbol{\phi}(\boldsymbol{x}) \mid \boldsymbol{\theta} \in \boldsymbol{\theta}^{I}\right\}
$$

with $\boldsymbol{\phi}$ a basis (e.g., polynomial or trigoniometric), $\boldsymbol{\theta}$ the fitting parameters of the IPM and $\boldsymbol{\theta}^{I}=[\underline{\boldsymbol{\theta}}, \overline{\boldsymbol{\theta}}]$ an $n_{\boldsymbol{\theta}}$-dimensional hyper-rectangular set. An optimal IPM is constructed by minimizing $E[(\overline{\boldsymbol{\theta}}-\underline{\boldsymbol{\theta}})|\boldsymbol{\phi}(\boldsymbol{x})|]$. Scenario Optimization [54] can be used to judge the generalization properties of the IPM. In case the corresponding optimization problem is convex, the reliability $R$ of the IPM (i.e., the probability that a future unobserved data point will be contained in the IPM) is bounded by:

$$
P(R \geq 1-\epsilon)>1-\beta,
$$

where $\epsilon$ and $\beta$ are the confidence and reliability parameters, which for our hyper-rectangular model can be obtained from

$$
\beta \geq\left(\begin{array}{c}
k+n_{\theta}-1 \\
k
\end{array}\right) \sum_{i=0}^{k+n_{\theta}-1}\left(\begin{array}{c}
N \\
i
\end{array}\right) \epsilon^{i}(1-\epsilon)^{N-i},
$$

where $k$ is the number of data points discarded by some algorithm and $\epsilon$ can be chosen as a very small number (e.g., $\epsilon=1 \cdot 10^{-06}$ ). An approach to apply IPMs in the context of propagating parametrized p-boxes is introduced by [102]. They show that IPMs can be used as surrogate model to speed up the calculation of Eq.(17) and Eq.(18), including a strategy to intelligently construct the set $\left\{\left(\boldsymbol{x}_{i}, \boldsymbol{y}_{i}\right) \mid i=\right.$ $1, \ldots, N\}$. Furthermore, they show that the IPM can also be used as a surrogate model for $g$, which in its turn can be used in combination with importance sampling to determine $[\underline{\mathcal{P}}, \overline{\mathcal{P}}]$. Other applications include estimating the bounds on $\mathcal{P}$ resulting from the surrogate model inaccuracy in a deterministic case [103].

The main advantages of these techniques are that (1) they are completely black-box as they don't require any assumption on $\mathcal{M}$ and (2) that under the mild assumption of convexity of the training guaranteed reliability bounds on the accuracy are obtained based on the rigorous framework of Scenario Optimization, which was recently extended to non-convex optimization problems too [54]. Unfortunately, active learning of this type of surrogate models is not feasible, since this violates the required assumptions on independence between the training samples [103]. 


\subsection{Concluding discussion}

As an attempt to create some clarity in the applicability of the multitude of available methods for the propagation of p-boxes, Table 2 summarizes the discussed methods, including their class, limitations and to which type of p-box they are applicable. Note that no precise statements on accuracy and/or numerical efficiency are given, as these depend fully on the problem under consideration. For instance, for linear models, the operator norm will undoubtedly give the best results from all 'direct' solution methods, as it reduces the solution of the problem to two deterministic optimization problems and two reliability analyses. On the other hand, for highly nonlinear problems, this method will fail, and potentially methods based on surrogate modelling will outperform the other methods. To make a fully fair comparison between these methods in this respect, a dedicated benchmark is study is required, which falls outside the scope of this paper. It should be noted, however, that in case there is no prerogative to use the numerical model, the computational efficiency of propagating imprecise probabilities with surrogate modelling approaches is orders of magnitude higher as compared to the approaches that directly use the numerical model. This is particularly true when advanced active learning methods such as AK-MCS [89] are applied in the context of reliability analysis.

Table 2: Summary of black-box propagation schemes for p-boxes

\begin{tabular}{|c|c|c|c|c|}
\hline Method & class & Type p-box & Limitation & ref \\
\hline Double loop & Direct & Both & Computational cost & \\
Interval Monte Carlo & Direct & Free & Computational cost & {$[62]$} \\
Random set methods & Direct & Both & Computational cost & {$[74]$} \\
Advanced Line Sampling & Decoupling & Param. & Moderate linearity & {$[80]$} \\
Extended Monte Carlo & Decoupling & Param. & Stochastic hyper-parameters & {$[39]$} \\
Operator norm & Decoupling & Param. & Linear models & {$[82]$} \\
PCE & Surrogate & Both & Global approximation of $\mathcal{M}$ & {$[57]$} \\
Kriging & Surrogate & Both & Local approximation of $\mathcal{M}$ & {$[104]$} \\
HDMR & Surrogate & Param. & Dimension of $\boldsymbol{x}$ & {$[100]$} \\
IPM & Surrogate & Param. & No adaptive refinement & {$[102]$} \\
\hline
\end{tabular}

Generally, optimization approaches such as double loop or sampling methods provide inner approximations of the bounds on $P_{f}$ as they generate realisations within $\left[\underline{F}_{X}(x), \bar{F}_{X}(x)\right]$ and try to approach $\underline{\mathcal{P}}$, respectively $\overline{\mathcal{P}}$ from the inside-out [43]. Note that, in case distribution-free p-box methods such as those based on random sets are applied to parametric p-boxes, this effectively constitutes an outer approximation.

\footnotetext{
${ }^{1}$ More general imprecise probability models can be considered too, please refer to Section 2.3 for more information
} 


\section{Conclusions}

The development of highly efficient approaches to perform engineering computations with imprecise probabilities, represented as p-boxes, is a quickly expanding field of research. The main challenge in this context is to overcome the required double loop propagation framework to estimate the bounds on probabilistic measures of the structure under consideration (such as, e.g., the probability of failure). Apart from near-trivial numerical simulation models, such double loop calculations are computationally intractable without resorting to high-performance computing facilities.

This problem is currently being tackled from two sides: (1) by improving the propagation efficiency of p-boxes aimed at breaking the double loop and (2) developing efficient surrogate models for the numerical models to be used in the double loop. Concerning the former set of solutions, highly efficient propagation schemes have been introduced in recent years. However, these methods are either limited in terms of the admissible descriptions of the uncertainty, or the non-linearity of the underlying numerical model. Future developments in these areas should concentrate on expanding the scope of applicability of these techniques. Concerning the latter, surrogate models usually only require some smoothness constraints on the underlying numerical model, which allows for a greater flexibility. Nonetheless, the accuracy of the calculation of the bounds on the probabilistic measures is limited to the accuracy of the underlying surrogate model. Furthermore, also the training of these surrogate models can entail a non-negligible numerical cost, which is commonly mitigated by resorting to active learning.

As such, to conclude, the last 15 years brought many highly performing approaches to compute with imprecise probabilities in general, and p-boxes in specific. The main challenge at this point appears to translate this set of highly performing methods to industrial applications involving multi-physical and/or million degree-of-freedom numerical models. Further work in this domain will include continuing developments on a theoretical side (e.g., constrained distribution-free p-boxes) and propagation aspects (e.g., operator norm theory for nonlinear dynamics). We expect that the current rapid developments in the domain of machine learning and big data can play a pivotal role in (1) the characterization of uncertainties, where the uncertainty characteristics are added by the machine learning algorithm, (2) the propagation and inverse identification of p-boxes, much alike active learning surrogate models, (3) performing dimension reduction by finding optimal representations of the uncertainty and (4) detecting dependencies in very high-dimensional datasets.

\section{Acknowledgements}

This paper presents an extended and revised version of the conference proceedings in [105]. Matthias Faes acknowledges the support of the Research Foundation Flanders (FWO) under grant number 12P3519N 
as well as the Alexander von Humboldt foundation. Marco Daub acknowledges the support of the German Academic Exchange Service (DAAD) with a postdoc fellowship.

\section{References}

[1] G. Schuëller, Computational Methods in Stochastic Dynamics, Springer Netherlands, 2011, pp. $1-24$.

[2] M. Faes, D. Moens, Recent Trends in the Modeling and Quantification of Non-probabilistic Uncertainty, Arch. Comput. Methods Eng. (2019).

[3] M. Beer, Y. Zhang, S. T. Quek, K. K. Phoon, Reliability analysis with scarce information: Comparing alternative approaches in a geotechnical engineering context, Struct. Saf. 41 (2013) 1-10.

[4] M. Broggi, M. Faes, E. Patelli, Y. Govers, D. Moens, M. Beer, Comparison of Bayesian and interval uncertainty quantification: Application to the AIRMOD test structure, in: 2017 IEEE Symposium Series on Computational Intelligence, SSCI 2017 - Proceedings, volume 2018-Janua, 2018, pp. 1-8.

[5] M. Faes, M. Broggi, E. Patelli, Y. Govers, J. Mottershead, M. Beer, D. Moens, A multivariate interval approach for inverse uncertainty quantification with limited experimental data, Mech. Syst. Sig. Process. 118 (2019) 534-548.

[6] G. Schuëller, H. Pradlwarter, Benchmark study on reliability estimation in higher dimensions of structural systems - An overview, Struct. Saf. 29 (2007) 167-182.

[7] K. Marti, Approximation and derivatives of probabilities of survival in structural analysis and design, Structural Optimization 13 (1997) 230-243.

[8] A. A. Taflanidis, J. Beck, Analytical approximation for stationary reliability of certain and uncertain linear dynamic systems with higher-dimensional output, Earthquake Engineering \& Structural Dynamics 35 (2006) 1247-1267.

[9] K. W. Breitung, Asymptotic Approximations for Probability Integrals, Springer, Berlin, 1994.

[10] S. Au, J. Beck, Estimation of small failure probabilities in high dimensions by subset simulation, Probab. Eng. Mech. 16 (2001) 263-277.

[11] M. A. Misraji, M. A. Valdebenito, H. A. Jensen, C. F. Mayorga, Application of directional importance sampling for estimation of first excursion probabilities of linear structural systems subject to stochastic Gaussian loading, Mech. Syst. Sig. Process. 139 (2020) 106621. 
[12] J. Li, J.-B. Chen, The probability density evolution method for dynamic response analysis of non-linear stochastic structures, Int. J. Numer. Methods Eng. 65 (2006) 882-903.

[13] M. Beer, S. Ferson, V. Kreinovich, Imprecise probabilities in engineering analyses, Mech. Syst. Sig. Process. 37 (2013) 4-29.

[14] W. L. Oberkampf, J. C. Helton, C. A. Joslyn, S. F. Wojtkiewicz, S. Ferson, Challenge problems: Uncertainty in system response given uncertain parameters, Reliability Engineering and System Safety 85 (2004) 11-19. doi:10.1016/j.ress.2004.03.002.

[15] M. H. Faber, On the Treatment of Uncertainties and Probabilities in Engineering Decision Analysis, Journal of Offshore Mechanics and Arctic Engineering 127 (2005) 243.

[16] A. D. Kiureghian, O. Ditlevsen, Aleatory or epistemic? Does it matter?, Structural Safety 31 (2009) 105-112.

[17] D. Veneziano, A. Agarwal, E. Karaca, Decision making with epistemic uncertainty under safety constraints: An application to seismic design, Probabilistic Engineering Mechanics 24 (2009) 426437.

[18] S. Bi, M. Broggi, M. Beer, The role of the Bhattacharyya distance in stochastic model updating, Mech. Syst. Sig. Process. 117 (2019) $437-452$.

[19] F. Tonon, A. Bernardini, A random set approach to the optimization of uncertain structures, Computers \& structures 68 (1998) 583-600.

[20] F. Tonon, Using random set theory to propagate epistemic uncertainty through a mechanical system, Reliability Engineering and System Safety 85 (2004) 169-181.

[21] D. Alvarez, J. Hurtado, J. Ramírez, Tighter bounds on the probability of failure than those provided by random set theory, Computers \& Structures 189 (2017) 101-113.

[22] T. Fetz, M. Oberguggenberger, Propagation of uncertainty through multivariate functions in the framework of sets of probability measures, Reliability Engineering and System Safety 85 (2004) 73-87. doi:10.1016/j.ress.2004.03.004.

[23] D. Dubois, H. Prade, Possibility Theory, volume 64, Springer US, Boston, MA, 1988. doi:10.1007/978-1-4684-5287-7.

[24] G. Shafer, A Mathematical Theory of Evidence turns 40, International Journal of Approximate Reasoning 79 (2016) 7-25. 
[25] J. C. Helton, J. D. Johnson, W. L. Oberkampf, An exploration of alternative approaches to the representation of uncertainty in model predictions, Reliability Engineering and System Safety 85 (2004) 39-71.

[26] W. L. McGill, B. M. Ayyub, Estimating parameter distributions in structural reliability assessment using the transferable belief model, Comput. Struct. 86 (2008) 1052-1060.

[27] T. Augustin, F. Coolen, G. De Cooman, M. Troffaes, Introduction to Imprecise Probabilities, John Wiley \& Sons Ltd, Chichester, 2014.

[28] V. Kreinovich, S. Ferson, A new Cauchy-based black-box technique for uncertainty in risk analysis, Rel. Eng. Syst. Saf. 85 (2004) 267-279.

[29] B. Möller, M. Beer, Fuzzy Randomness, Springer, Berlin, 2004. URL: http://link.springer.com/10.1007/978-3-662-07358-2. doi:10.1007/978-3-662-07358-2.

[30] M. Beer, M. Zhang, S. Quek, S. Ferson, Structural reliability assessment with fuzzy probabilities, in: [30], 2011.

[31] A. Decadt, G. de Cooman, J. De Bock, Monte Carlo Estimation for Imprecise Probabilities: Basic Properties (2019). URL: http://arxiv.org/abs/1905.09301. arXiv:1905.09301.

[32] M. Troffaes, Imprecise monte carlo simulation and iterative importance sampling for the estimation of lower previsions, Int. J. Approximate Reasoning 101 (2018) $31-48$.

[33] J. W. Hall, Uncertainty-based sensitivity indices for imprecise probability distributions, Reliability Engineering \& System Safety 91 (2006) 1443-1451.

[34] S. Ferson, V. Kreinovich, L. Ginzburg, D. S. Myers, K. Sentz, Constructing Probability Boxes and Dempster-Shafer Structures, Technical Report January, Technical report, Sandia National Laboratories, 2003.

[35] W. L. Oberkampf, W. T. Tucker, J. Zhang, L. Ginzburg, D. J. Berleant, S. Ferson, J. Hajagos, R. B. Nelsen, Dependence in probabilistic modeling, Dempster-Shafer theory, and probability bounds analysis., Technical Report, Sandia National Laboratories (SNL), Albuquerque, NM, and Livermore, CA, 2004. URL: http://www.osti.gov/servlets/purl/919189-POVF66/. doi:10.2172/919189.

[36] I. Montes, E. Miranda, Bivariate p-boxes and maxitive functions, International Journal of General Systems 46 (2017) 354-385. URL: 
https://www.tandfonline.com/doi/full/10.1080/03081079.2017.1305960. doi:10.1080/03081079.2017.1305960.

[37] M. Dannert, A. Fau, R. Fleury, M. Broggi, U. Nackenhorst, M. Beer, A probability-box approach on uncertain correlation lengths by stochastic finite element method, PAMM (Proceedings in Applied Mathematics and Mechanics) 18 (2018) e201800114.

[38] M. Faes, D. Moens, Imprecise random field analysis with parametrized kernel functions, Mech. Syst. Sig. Process. 134 (2019) 106334.

[39] P. Wei, J. Song, S. Bi, M. Broggi, M. Beer, Z. Lu, Z. Yue, Non-intrusive stochastic analysis with parameterized imprecise probability models: I. performance estimation, Mech. Syst. Sig. Process. $124(2019) 349-368$.

[40] P. Wei, J. Song, S. Bi, M. Broggi, M. Beer, Z. Lu, Z. Yue, Non-intrusive stochastic analysis with parameterized imprecise probability models: II. reliability and rare events analysis, Mech. Syst. Sig. Process. 126 (2019) $227-247$.

[41] M. G. R. Faes, M. A. Valdebenito, X. Yuan, P. Wei, M. Beer, Augmented Reliability Analysis for Estimating Imprecise First Excursion Probabilities in Stochastic Linear Dynamics, preprint submitted to elsevier (2020).

[42] D. A. Alvarez, On the calculation of the bounds of probability of events using infinite random sets, International Journal of Approximate Reasoning 43 (2006) 241-267. doi:10.1016/j.ijar.2006.04.005.

[43] E. Patelli, D. A. Alvarez, M. Broggi, M. de Angelis, Uncertainty Management in Multidisciplinary Design of Critical Safety Systems, Journal of Aerospace Information Systems 12 (2014) 140-169.

[44] D. A. Alvarez, J. E. Hurtado, J. Ramírez, Tighter bounds on the probability of failure than those provided by random set theory, Computers and Structures 189 (2017) 101-113.

[45] B. Möller, M. Beer, Fuzzy Randomness, Springer Berlin Heidelberg, Berlin, Heidelberg, 2004. URL: http://link. springer. com/10.1007/978-3-662-07358-2. doi:10.1007/978-3-662-07358-2.

[46] V. J. Montgomery, F. P. A. Coolen, A. D. M. Hart, Bayesian Probability Boxes in Risk Assessment, Journal of Statistical Theory and Practice 3 (2009) 6983. URL: http://www.tandfonline.com/doi/abs/10.1080/15598608.2009.10411912. doi:10.1080/15598608.2009.10411912. 
[47] R. Schöbi, Surrogate models for uncertainty quantification in the context of imprecise probability modelling, Doctoral thesis, ETH Zürich, Switzerland, 2017. doi:https://doi.org/10.3929/ethz-a010870825 .

[48] M. Oberguggenberger, W. Fellin, Reliability bounds through random sets: Nonparametric methods and geotechnical applications, Computers \& Structures 86 (2008) 1093-1101. URL: https://linkinghub.elsevier.com/retrieve/pii/S0045794907002192. doi:10.1016/j.compstruc.2007.05.040.

[49] M. Troffaes, T. Basu, A cantelli-type inequality for constructing non-parametric p-boxes based on exchangeability, volume 103 of Proceedings of Machine Learning Research, PMLR, Thagaste, Ghent, Belgium, 2019, pp. 386-393. URL: http://proceedings.mlr.press/v103/troffaes19a.html.

[50] M. Oberguggenberger, W. Fellin, Reliability bounds through random sets: Non-parametric methods and geotechnical applications, Computers and Structures 86 (2008) 1093-1101. doi:10.1016/j.compstruc.2007.05.040.

[51] J. G. Saw, M. C. K. Yang, T. C. Mo, Chebyshev Inequality with Estimated Mean and Variance, The American Statistician 38 (1984) 130. URL: https://www . jstor .org/stable/2683249?origin=crossref. doi:10.2307/2683249.

[52] L. G. Crespo, B. K. Colbert, S. P. Kenny, D. P. Giesy, On the quantification of aleatory and epistemic uncertainty using Sliced-Normal distributions, Systems \& Control Letters 134 (2019) 104560.

[53] M. Imholz, M. Faes, D. Vandepitte, D. Moens, Robust uncertainty quantification in structural dynamics under scarse experimental modal data: A bayesian-interval approach, J. Sound Vib. 467 (2020) 114983.

[54] M. C. Campi, S. Garatti, F. A. Ramponi, A General Scenario Theory for Nonconvex Optimization and Decision Making, IEEE Trans. Autom. Control 63 (2018) 4067-4078.

[55] A. Kolmogoroff, Confidence Limits for an Unknown Distribution Function, The Annals of Mathematical Statistics 12 (1941) 461-463. URL: http://projecteuclid.org/euclid.aoms/1177731684. doi:10.1214/aoms/1177731684.

[56] J. O. Berger, Statistical Decision Theory and Bayesian Analysis, Springer Series in Statistics, Springer New York, New York, NY, 1985. doi:10.1007/978-1-4757-4286-2. 
[57] R. Schöbi, B. Sudret, Structural reliability analysis for p-boxes using multi-level meta-models, Probab. Eng. Mech. 48 (2017) 27-38.

[58] T. Aldenberg, J. S. Jaworska, Uncertainty of the Hazardous Concentration and Fraction Affected for Normal Species Sensitivity Distributions, Ecotoxicology and Environmental Safety 46 (2000) 1-18. URL: https://linkinghub.elsevier.com/retrieve/pii/S0147651399918699. doi:10.1006/eesa.1999.1869.

[59] L. Held, D. Sabanés Bové, Likelihood and Bayesian Inference, Statistics for Biology and Health, Springer Berlin Heidelberg, Berlin, Heidelberg, 2020. URL: http://link. springer.com/10.1007/978-3-662-60792-3. doi:10.1007/978-3-662-60792-3.

[60] X. Liu, Z. Kuang, L. Yin, L. Hu, Structural reliability analysis based on probability and probability box hybrid model, Structural Safety 68 (2017) 73-84. doi:10.1016/j.strusafe.2017.06.002.

[61] C. Wang, H. Zhang, M. Beer, Computing tight bounds of structural reliability under imprecise probabilistic information, Computers \& Structures 208 (2018) 92 - 104.

[62] H. Zhang, R. L. Mullen, R. L. Muhanna, Interval Monte Carlo methods for structural reliability, Struct. Saf. 32 (2010) 183-190.

[63] H. Zhang, H. Dai, M. Beer, W. Wang, Structural reliability analysis on the basis of small samples: An interval quasi-Monte Carlo method, Mech. Syst. Sig. Process. 37 (2013) 137-151.

[64] Z. P. Qiu, I. Elishakoff, Anti-optimization of structures with large uncertain-but-non-random parameters via interval analysis, Computer Methods in Applied Mechanics and Engineering 152 (1998) 361-372.

[65] M. Zettler, J. Garloff, Robustness analysis of polynomials with polynomial parameter dependency using Bernstein expansion, IEEE Transactions on Automatic Control 43 (1998) 425-431.

[66] L. G. Crespo, S. P. Kenny, D. P. Giesy, Reliability analysis of polynomial systems subject to p-box uncertainties, Mechanical Systems and Signal Processing 37 (2013) 121-136.

[67] A. C. Calder, M. M. Hoffman, D. E. Willcox, M. P. Katz, F. D. Swesty, S. Ferson, Quantification of Incertitude in Black Box Simulation Codes, Journal of Physics: Conference Series 1031 (2018).

[68] M. De Angelis, S. Ferson, E. Patelli, V. Kreinovich, Black-box propagation of failure probabilities under epistemic uncertainty, Proceedings of the 3rd International Conference on Uncertainty Quantification in Computational Sciences and Engineering, UNCECOMP 2019 (2019) 713-723. 
[69] M. Hanss, Applied Fuzzy Arithmetic, Springer Berlin Heidelberg, 2005.

[70] M. Neher, From Interval Analysis to Taylor Models - An Overview, International Association for Mathematics and Computers in Simulation (2005) 102-110.

[71] J. A. Enszer, Y. Lin, S. Ferson, G. F. Corliss, M. A. Stadtherr, Probability bounds analysis for nonlinear dynamic process models, AIChE Journal 57 (2011) 404-422.

[72] N.-C. Xiao, Y.-F. Li, L. Yu, Z. Wang, H.-Z. Huang, Saddlepoint approximation-based reliability analysis method for structural systems with parameter uncertainties, Proceedings of the Institution of Mechanical Engineers, Part O: Journal of Risk and Reliability 228 (2014) 529-540.

[73] D. A. Alvarez, A Monte Carlo-based method for the estimation of lower and upper probabilities of events using infinite random sets of indexable type, Fuzzy Sets and Systems 160 (2009) 384-401.

[74] D. A. Alvarez, F. Uribe, J. E. Hurtado, Estimation of the lower and upper bounds on the probability of failure using subset simulation and random set theory, Mechanical Systems and Signal Processing 100 (2018) 782-801.

[75] X. Du, Unified uncertainty analysis by the first order reliability method, Journal of Mechanical Design, Transactions of the ASME 130 (2008) 0914011-09140110. doi:10.1115/1.2943295.

[76] J. Sadeghi, M. De Angelis, E. Patelli, Analytic Probabilistic Safety Analysis under Severe Uncertainty, ASCE-ASME Journal of Risk and Uncertainty in Engineering Systems, Part A: Civil Engineering 6 (2020). doi:10.1061/AJRUA6.0001028.

[77] P. Wei, Z. Lu, J. Song, Extended Monte Carlo simulation for parametric global sensitivity analysis and optimization, AIAA Journal 52 (2014) 867-878.

[78] J. Zhang, M. D. Shields, On the quantification and efficient propagation of imprecise probabilities resulting from small datasets, Mech. Syst. Sig. Process. 98 (2018) 465-483.

[79] J. Zhang, M. D. Shields, Efficient Monte Carlo resampling for probability measure changes from Bayesian updating, Probab. Eng. Mech. 55 (2019) 54-66.

[80] M. de Angelis, E. Patelli, M. Beer, Advanced line sampling for efficient robust reliability analysis, Struct. Saf. 52, Part B (2015) 170-182.

[81] P. Koutsourelakis, H. Pradlwarter, G. Schuëller, Reliability of structures in high dimensions, part I: Algorithms and applications, Probab. Eng. Mech. 19 (2004) 409-417. 
[82] M. Faes, M. A. Valdebenito, D. Moens, B. Michael, Bounding the First Excursion Probability of Linear Structures Subjected to Imprecise Stochastic Loading, preprint submitted to elsevier (2020).

[83] J. A. Tropp, Topics in Sparse Approximation, Ph.D. thesis, The University of Texas at Austin, 2004.

[84] T. Soong, M. Grigoriu, Random Vibration of Mechanical and Structural Systems, Prentice Hall, Englewood Cliffs, New Jersey, 1993.

[85] K. Marti, Differentiation of probability functions: The transformation method, Computers \& Mathematics with Applications 30 (1995) 361-382.

[86] Y. Zhang, A. Der Kiureghian, First-excursion probability of uncertain structures, Probab. Eng. Mech. 9 (1994) 135-143.

[87] M. G. R. Faes, M. A. Valdebenito, Fully Decoupled Reliability-Based Design Optimization of Structural Systems Subject to Uncertain Loads, Computer Methods in Applied Mechanics and Engineering (2020).

[88] S. Marelli, B. Sudret, An active-learning algorithm that combines sparse polynomial chaos expansions and bootstrap for structural reliability analysis, Struct. Saf. 75 (2018) $67-74$.

[89] N. Lelièvre, P. Beaurepaire, C. Mattrand, N. Gayton, AK-MCSi: A Kriging-based method to deal with small failure probabilities and time-consuming models, Struct. Saf. 73 (2018) 1 - 11.

[90] J. Zhang, M. Xiao, L. Gao, An active learning reliability method combining kriging constructed with exploration and exploitation of failure region and subset simulation, Rel. Eng. Syst. Saf. 188 (2019) $90-102$.

[91] A. Sofi, G. Muscolino, F. Giunta, Propagation of uncertain structural properties described by imprecise Probability Density Functions via response surface method, Probab. Eng. Mech. (2020) 103020.

[92] W. Gao, D. Wu, C. Song, F. Tin-Loi, X. Li, Hybrid probabilistic interval analysis of bar structures with uncertainty using a mixed perturbation monte-carlo method, Finite Elem. Anal. Des. 47 (2011) 643-652.

[93] P. Angelikopoulos, C. Papadimitriou, P. Koumoutsakos, X-TMCMC: Adaptive kriging for Bayesian inverse modeling, Computer Methods in Applied Mechanics and Engineering 289 (2015) 409-428. doi:10.1016/j.cma.2015.01.015. 
[94] C. Ling, Z. Lu, X. Zhu, Efficient methods by active learning kriging coupled with variance reduction based sampling methods for time-dependent failure probability, Rel. Eng. Syst. Saf. 188 (2019) 23 -35 .

[95] S. Marelli, B. Sudret, An active-learning algorithm that combines sparse polynomial chaos expansions and bootstrap for structural reliability analysis, Structural Safety 75 (2018) 67-74. doi:https://doi.org/10.1016/j.strusafe.2018.06.003.

[96] R. Schöbi, B. Sudret, Uncertainty propagation of p-boxes using sparse polynomial chaos expansions, J. Comput. Phys. 339 (2017) 307-327.

[97] H. B. Liu, C. Jiang, Z. Xiao, Efficient uncertainty propagation for parameterized p-box using sparse-decomposition-based polynomial chaos expansion, Mech. Syst. Sig. Process. 138 (2020) 106589 .

[98] N.-C. Xiao, K. Yuan, C. Zhou, Adaptive Kriging-based efficient reliability method for structural systems with multiple failure modes and mixed variables, Comput. Methods Appl. Mech. Eng. 359 (2020) 112649.

[99] J. Song, M. Valdebenito, P. Wei, M. Beer, Z. Lu, Non-intrusive imprecise stochastic simulation by line sampling, Struct. Saf. 84 (2020) 101936.

[100] M. Fina, P. Weber, W. Wagner, Polymorphic uncertainty modeling for the simulation of geometric imperfections in probabilistic design of cylindrical shells, Struct. Saf. 82 (2020) 101894.

[101] L. G. Crespo, S. P. Kenny, D. P. Giesy, Interval Predictor Models With a Linear Parameter Dependency, Journal of Verification, Validation and Uncertainty Quantification 1 (2016) 1-10.

[102] J. Sadeghi, M. de Angelis, E. Patelli, Robust propagation of probability boxes by interval predictor models, Struct. Saf. 82 (2020) 101889.

[103] M. Faes, J. Sadeghi, M. Broggi, M. de Angelis, E. Patelli, M. Beer, D. Moens, On the Robust Estimation of Small Failure Probabilities for Strong Nonlinear Models, ASCE-ASME J Risk and Uncert in Engrg Sys Part B Mech Engrg 5 (2019).

[104] R. Schöbi, B. Sudret, Global sensitivity analysis in the context of imprecise probabilities (p-boxes) using sparse polynomial chaos expansions, Rel. Eng. Syst. Saf. 187 (2019) 129 - 141. Sensitivity Analysis of Model Output. 
[105] M. G. R. Faes, M. Daub, M. Beer, Engineering analysis with imprecise probabilities: a state-of-theart review on p-boxes, Proceedings of the 7th Asian-Pacific Symposium on Structural Reliability and its Applications, APSSRA 2020 (2020). 